\title{
Erratum: Polarization of active Janus particles [Phys. Rev. E 89, 050303(R) (2014)]
}

Thomas Bickel, Guillermo Zecua, and Alois Würger

(Received 4 April 2018; published 24 April 2018)

DOI: 10.1103/PhysRevE.97.049901

We became aware that the term proportional to $\mu^{\prime}+\mu$ in Eq. (8b) of this paper is not correct. This equation should read

$$
\mathbf{A}_{0}=-\tau\left(\mu^{\prime}-\mu\right) \frac{5}{18} \xi_{1} \frac{3 Q_{0}}{8 \kappa_{s}} .
$$

This error stems from a missing factor $(\sin \theta)^{-1}$ in the azimuthal term of Eq. (SM 45), and concerns the calculations in Sec. V C of the Supplemental Material where the even coefficients in Eq. (SM 46) should read $k_{2 n}=0$, resulting in $\tilde{\xi}=0$ in Eqs. (SM 47) and (SM 48).

In the main paper, this error affects only Eq. (8b); in view of the corrected expression, the two sentences following (8b) are rather meaningless. The rest of the paper, including the abstract, discussion, and conclusions, remains valid. In particular, the terms proportional to $\mu+\mu^{\prime}$ in the linear velocities in Eqs. (2), (7), and (13) are correct.

We thank M. Yang for helpful remarks. 